\title{
Role of MRI Diffusion in Differentiation between Benign and Malignant Parotid Gland Tumors
}

\author{
Medhat M. Rafat, Islam M. El- shazly, Christen h. mekhael
}

\begin{abstract}
Department of Radiology, Benha faculty of medicine, Benha University, Egypt

Correspondence to: Christen h. mekhael, Department of Radiology, Benha faculty of medicine, Benha University, Egypt .
\end{abstract}

Email:

christen.helmy@su.edu.eg

Received: 21 April 2021

Accepted: 5 May 2021

\begin{abstract}
:
Background: The potential additional value of diffusion in oncological imaging lies in the fact that it provides functional tissue information, which can be combined with anatomical MR images to improve the specificity of lesion characterization.The DWI studies and ADC measurements provide useful information about tumor cellularity that can correlate with the histological pattern of parotid lesion and give a clue in differentiation between benign and malignant tumors. Aim of work: this work aims to study the value of the diffusion weighted imaging and ADC measurement coupled with a high-resolution MR imaging technique that can enable effective characterization of histologic features of the parotid tumors. We also expected that mapping of the ADC could differentiate benign from malignant tumors. Patients and methods: This study included 25 (14female and11 male) . Patients ages ranged from 3-69 year old. All the patients presented to the "National Cancer Institute" during the past year. Results :combining DWI and ADC values has increased accuracy, as the sensitivity and specificity were $92.86 \%, 90.91 \%$ respectively with $92.86 \%$ positive predictive value and $90.91 \%$ negative predicative value Conclusion :our date and in agreement with previous studies had demonstrated that determining whether a parotid gland tumor is benign or malignant on the basis of combining morphologic features and DWIs \& ADC measurements in a diagnostic noninvasive approach are very promising and might decrease number of unnecessary
\end{abstract} invasive procedures

Keywords: MRI diffusion, ADC , parotid tumors 


\section{Introduction}

Parotid glands are involved by a wide variety of different tumor subtypes. Understanding of these pathologic processes with correct preoperative diagnosis is required for proper surgical planning

Appropriate surgical approach depends on the preoperative differentiation between benign and malignant lesions in addition to the determination of the exact histologic subtypes. Furthermore, the reported risk for local recurrence varies with the tumor subtype.

Fine-needle aspiration cytology (FNAC) which provides a cytological diagnosis is a minimally invasive, reliable procedure, easy to perform and is widely used for evaluation of parotid masses and surgical planning. However, the most significant problem with FNAC is that the procedure frequently obtains inadequate material, thus making evaluation impossible and it carries a risk of tumor spread that can lead to a possible recurrence. Additionally, it is proved that intraoperative frozen section analysis is superior to FNAC.

Pre-operative diagnosis of the type of parotid mass can help the surgeon determine the most suitable surgical procedure. MRI is a noninvasive technique that can provide morphological information of the mass, allow correct diagnosis and proper staging. It can accurately determine the deep or superficial location of the parotid mass, extension, contour and signal pattern.

Diffusion-weighted imaging (DWI) provides functional information related to the random water diffusion of the mass and has a high ability to determine different histologic subtypes of parotid tumors. Apparent diffusion coefficient (ADC) values calculated from DWI can provide quantitative information related to random diffusion of water molecules in tissues and functionally complement conventional MRI and has been reported as helpful for narrowing the differential diagnosis of parotid masses.

The purpose of this prospective study is to investigate the potential application of new MRI techniques with emphasis to diffusion weighted echo planar imaging (DW-EPI) in characterization and differentiation of various entities of parotid gland tumors and to correlate diffusion patterns \& ADC 
values of different parotid lesions with their pathological nature.

\section{Aim of work}

To investigate the potential application of new MRI techniques with emphasis to diffusion weighted echo planar imaging (DW-EPI) in characterization and differentiation of various entities of parotid gland tumors and to correlate diffusion patterns \& ADC values of different parotid lesions with their pathological nature.

\section{Patients and methods}

Study Population

This prospective descriptive analytic study included 25 (14 female and 11 male) patients. Patients ages ranged from $3-69$ year with a mean of $33.14 \pm 22.40$ (mean \pm SD). All the patients presented to the "National Cancer Institute" during the past year.

Comprehensive explanations of the procedures were done for all cases including the associated risks and contraindications.

Inclusion criteria

- Patient with parotid or pre-auricular swellings.
- Patients who had clinical and radiological findings suggestive of parotid neoplasm.

Exclusion criteria

- Patients with no parotid lesions.

- Patients with a contraindication to MRI e.g. claustrophopic, applied cardiac pacemaker or cochlear implants and contraindication to IV contrast administration e.g. renal impairment.

Patient preparation:

Detachable metallic implants like teeth prosthesis are considered as relative contraindication should be removed prior to entrance to magnetic area.

For the patient needed anathesthia; fasting four hours before the scan is required.

Technique:-

\section{Conventional MRI}

All the patient were evaluated by MRI technique using 1.5 Tesla superconducting MR imager (Philips Achieva, Best, Netherlands)

All the cases were examined in supine position with standard circularly polarized head coil using the following sequences and parameters:- 
- Axial T1WI TR/TE (659/12 ms) spin echo.

- Axial T2WI TR/TE (3680/93 ms) spin echo.

- Axial STIR TR/TE/TI (9000 / 116/ 2500 $\mathrm{ms})$.

- Coronal T1WI TR/TE (430 / 10 ms) spin echo.

- Coronal T2 TR/TE (3680/93 ms) spin echo.

- 256 x 256 matrix.

- Section thickness $3 \mathrm{~mm}$ for axial cuts and $4 \mathrm{~mm}$ for coronal cuts.

- Field of view $280 \mathrm{~mm}$ for axial cuts and $340 \mathrm{~mm}$ for coronal cuts.

- Resolution 512.

- Voxel size 1.1 × 0.5 × $3.0 \mathrm{~mm}$ for axial cuts and $1.3 \times 0.7 \times 4.0 \mathrm{~mm}$.for coronal cuts.

After intravenous administration of Gadolinium-DTPA $(0.3 \mathrm{mg} / \mathrm{kg})$, contrast enhanced T1WI (TR, 693 ms; TE, 12 $\mathrm{ms}$ ) in axial \pm fat suppression, sagittal and coronal planes were obtained.

The lesions were evaluated in conventional MRI commenting on:
- The side of the lesion "uni or bilateral".

- Site of the lesion "superficial lobe, deep lobe or both".

- The number of the lesions if more than one.

- Signal behavior on T1, T2 WIs and STIR.

- Heterogenity (presence of intratumoral hemorrhagic or necrotic components)

- Enhancement degree and pattern.

- Presence of lymph nodes and their levels.

- Vascular encasement of the external carotid artery or retromandibular vein or the carotid sheath in large lesions extending posteriorly.

- Facial nerve involvement "i.e. intraparotid segment".

- Perineural spread through the course of facial nerve "i.e. stylomastoid tunnel" or auriculo-temporal nerve "branch of Mandibular nerve V3".

- Bone invasion "e.g. mandible or skull base".

2. MR Diffusion Weighted Imaging:

An axial EPI DWI sequence with the following parameters: 
- TR, $1500 \mathrm{~ms}$; TE, $77 \mathrm{~ms}$.

- Matrix of 119 x 128.

- An FOV of 250 x $250 \mathrm{~mm}$ (pixel size 2.10 x $1.95 \mathrm{~mm}), 6$ excitations.

- A section thickness of $5 \mathrm{~mm}$ with an intersection gap of $1 \mathrm{~mm}$.

- A parallel imaging technique (modified sensitivity encoding) with an acceleration factor of 2 with 12 additional lines for selfcalibrating was applied.

- A bandwidth of $1502 \mathrm{~Hz} /$ pixel was used and 12 sections were acquired.

- The $\mathrm{b}$ factors used were $0 \mathrm{~s} / \mathrm{mm} 2,500$ $\mathrm{s} / \mathrm{mm} 2$ and $1000 \mathrm{~s} / \mathrm{mm} 2$.

Fat suppression was achieved by placing the frequency-selective radio-frequency pulse before the pulse sequence. The automatic 3D shim routine of the magnet used the section block as the shim volume and shimming was performed directly before the sequence was started. The total acquisition time of this sequence was 1 minute 14 seconds.

For each epi DWI sequence, a pixel-bypixel apparent diffusion coefficient (ADC) map was automatically calculated, with the gray value of the pixel linearly corresponding to the ADC value expressed in square millimeters per second.

\section{Results:}

Locations of the lesions

$32 \%$ of lesions were found in the right parotid gland, $56 \%$ were found in the left parotid gland and 12\% were found bilaterally in both parotid glands.

$16 \%$ of the studied lesions were found in the deep lobe, $56 \%$ were found in the superficial lobe while $28 \%$ of lesions existed in both lobes

Pathological diagnosis:

Patients where distributed according to final pathological diagnosis into 2 groups; the benign group and the malignant group. The benign group included 11patients while the malignant group included 14 patients

The diagnoses in the benign group as determined by pathological analysis were diverse: pleomorphic adenoma, warthin tumor, haemangioma, lymphangioma, basal cell adenoma and others as documented in the "Table. 11". Pleomorphic adenoma was the most frequent among benign lesions representing $36.36 \%$ of all benign lesions. 
Whereas malignant group showed the following entities: mucoepidermoid carcinoma, adenoid cystic carcinoma (ADCC), basal cell carcinoma, mixed salivary gland tumor, rabdomyosarcoma, lymphoma, leukemic infiltrates, metastatic intra-parotidlesionsand others. Even rare cases were found such as primitive neuro ectodermal tumor (PNET) "Table. 11".

However the most common malignant lesions found in our study were metastatic lesions to the parotid gland "i.e. direct infiltration or distant metastasis" representing $28.57 \%$; while the most common primary parotid tumor was found to be lymphoma representing about $9.09 \%$ in all malignant lesions(Fig. 16).

using conventional MRI alone in predicting benign and malignant lesions has the sensitivity of $68.75 \%$ ("CI"60.55\% to $87.95 \%)$ and specificity of $66.67 \%(95 \%$ CI $31.30 \%$ to $68.70 \%)$ with $78.57 \%$ (95\% CI $52.88 \%$ to $80.91 \%$ ) positive predictive value and $54.55 \%(95 \%$ CI $38.67 \%$ to $78.87 \%$ ) negative predicative value.

The lesions were assessed again, this time combined conventional MRI findings with DWI considering the DWI signal and ADC value.

After combination of conventional MRI and DWI;14 malignant looking lesions were detected, 13 proved to be true malignant lesions and one false positive. As 11 benign looking ones were detected, 10 proved positive with one false negative "Table. 27".

So, combining DWI and ADC values has increased accuracy, as the sensitivity and specificity were $92.86 \%$ (95\% CI $87.71 \%$ to $99.94 \%$ ), $90.91 \%$ (95\% CI $82.78 \%$ to $99.92 \%)$ respectively with $92.86 \%(95 \%$ CI $87.71 \%$ to $99.94 \%$ ) positive predictive value and $90.91 \%(95 \% \mathrm{CI} 82.78 \%$ to 99.92\%) negative predicative value(Table. 28 and Fig. 21).

Table (1): Showing all detected pathologies in benign and malignant groups.

\begin{tabular}{lcc}
\hline Pathological lesion & Frequency & Percentage \\
\hline Pleomorphic Adenoma & 4 & $36.36 \%$ \\
Chronic nonspecific inflammation & 2 & $18.18 \%$ \\
Warthin tumor & 2 & $18.18 \%$ \\
Lymphangioma & 1 & $9.09 \%$ \\
Basal cell adenoma & 1 & $9.09 \%$ \\
Non sebaceous lymphadenoma & 1 & $9.09 \%$ \\
\hline
\end{tabular}




\begin{tabular}{|c|c|c|c|}
\hline Malignant & Pathological lesion & Frequency & Percentage \\
\hline & Metastatic lesions & 4 & $28.57 \%$ \\
\hline & AdCC & 1 & $7.14 \%$ \\
\hline & Lymphoma & 3 & $21.42 \%$ \\
\hline & Leukemia & 1 & $7.14 \%$ \\
\hline & RMS & 1 & $7.14 \%$ \\
\hline & mucoepidermoid carcinoma & 1 & $7.14 \%$ \\
\hline & Basal cell carcinoma & 1 & $7.14 \%$ \\
\hline & $\begin{array}{l}\text { Mixed salivary gland } \\
\text { carcinoma }\end{array}$ & 1 & $7.14 \%$ \\
\hline & $\begin{array}{l}\text { poorly differentiated round } \\
\text { cell tumor }\end{array}$ & 1 & $7.14 \%$ \\
\hline
\end{tabular}

Table (2): Showing benign and malignant looking lesions characterized by combined conventional MRI and DWI.

\begin{tabular}{lcc}
\hline & Frequency & Percentage \\
\hline Benign & 11 & $44 \%$ \\
Malignant & 14 & $56 \%$ \\
\hline
\end{tabular}

Table (3): Showing sensitivity, specificity, positive predictive value and negative predictive value of the combined MRI and DWIs.

\begin{tabular}{lll}
\hline Statistic measure & Value & $\mathbf{9 5 \%}$ CI \\
\hline Sensitivity & $92.86 \%$ & $87.71 \%$ to $99.94 \%$ \\
Specificity & $90.91 \%$ & $82.78 \%$ to $99.92 \%$ \\
Positive Predictive Value & $92.86 \%$ & $87.71 \%$ to $99.94 \%$ \\
Negative Predictive Value & $90.91 \%$ & $82.78 \%$ to $99.92 \%$ \\
\hline
\end{tabular}




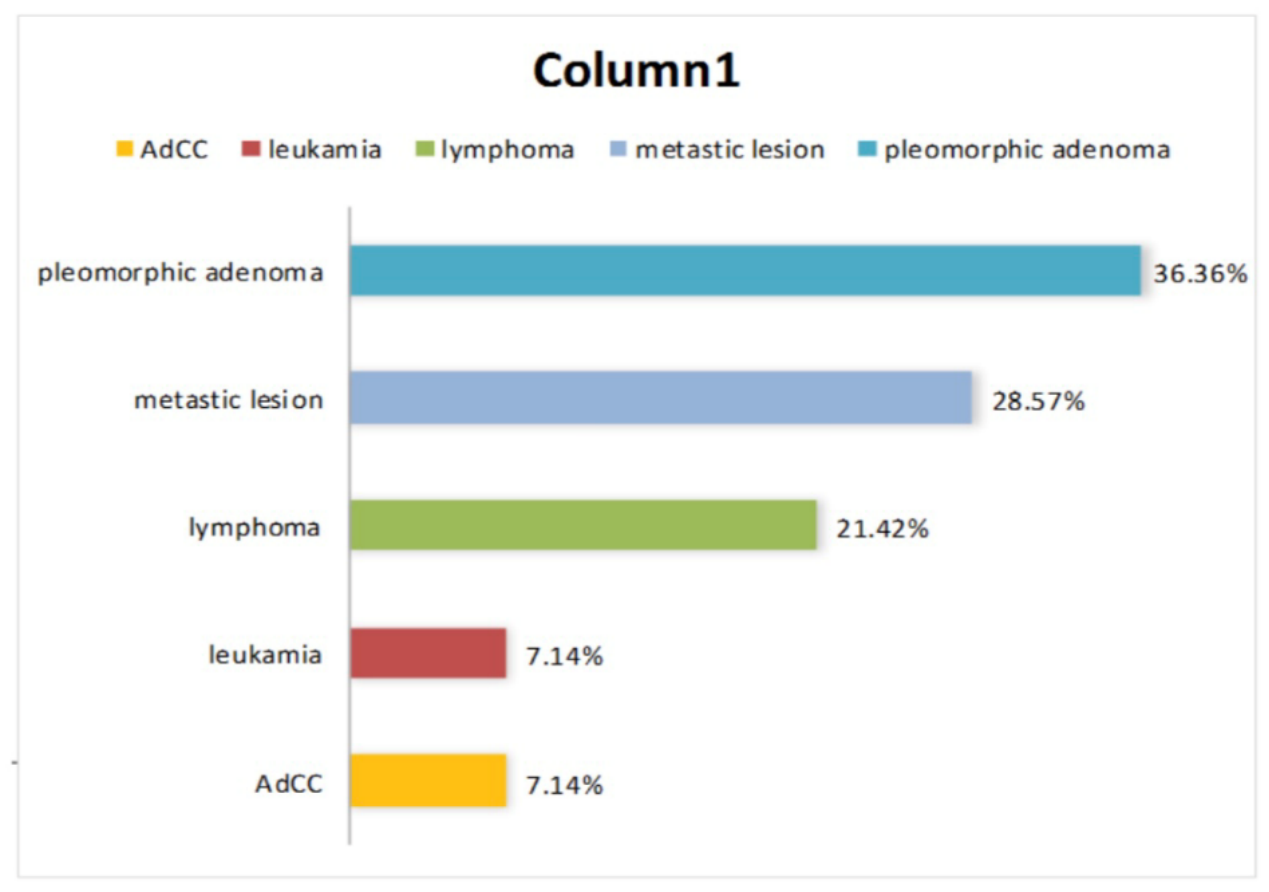

Figure (1): Bar chart showing most common malignant lesions in our study representing more than $60 \%$ of cases.

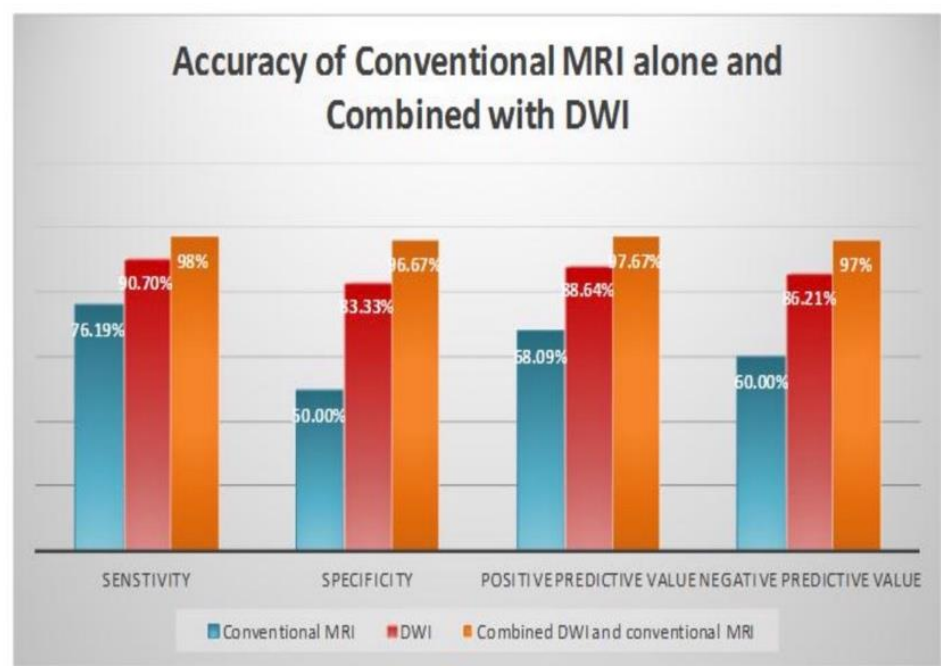

Figure (2): Showing accuracy measures; sensitivity, specificity, negative and positive predictive values; using conventional MRI alone, DWI alone and combined MRI with diffusion 


\section{Discussion}

Parotid gland tumors represent majority of salivary gland tumors which account for about $3 \%$ of all head and neck cancers

Preoperative discrimination between benign and malignant parotid gland tumors is a critical point and strongly influences the approach of surgical management. MR imaging has been established as a method for evaluating parotid gland lesions. Conventional T1- and T2-weighted MR imaging techniques with or without gadolinium enhancement have been a powerful tool for determining the morphology and extent of parotid gland tumors as well as their relationship to the surrounding structures .

Parotid gland tumors can be often diagnosed by fine needle aspiration cytology "FNAC", yet these minimally invasive procedures entails the risk of tumor spillage and inconclusive results owing to insufficient samples for histopathological analyses. Thus preoperative imaging plays an important role in the evaluation of tumor characteristics .

Patients were distributed according to the final pathological diagnosis into 2 groups; the benign group and the malignant group.
The benign group included 11patients while the malignant group included 14 patients.

In our study, $32 \%$ of lesions were found in the right parotid gland, $56 \%$ were found in the left parotid gland and $12 \%$ were found bilaterally in both parotid glands.

Also, $16 \%$ of the studied lesions were found in the deep lobe, $56 \%$ were found in the superficial lobe while $28 \%$ of lesions existed in both lobes.

The diagnosis in the benign group was determined by pathological analysis: pleomorphic adenoma (4 cases 36.36\%), Warthin tumor(2 cases 18.18\%), lymphangioma (1 case $9.09 \%$ ), basal cell adenoma(1 case $9.09 \%)$, non-sebaceous lymphadenoma (1 case 9.09\%) and chronic nonspecific inflammation (2 cases18.18\%). Pleomorphic adenoma showed to the most frequent among benign lesions representing $36.36 \%$ of all benign lesions.

Whereas malignant group shows the following entities: metastatic intra-parotid lesions (4 cases 28.57\%), lymphoma (3 cases $21.42 \%$ ), mucoepidermoid carcinoma (1 case $7.14 \%$ ), AdCC (1 case $7.14 \%$ ), basal cell carcinoma (1 case $7.14 \%)$, mixed salivary gland tumor(1 case $7.14 \%$ ), 
rabdomyosarcoma (1 case $7.14 \%$ ), leukemic infiltrates (1 case 7.14\%) and poorly differentiated round cell tumour (1 case $7.14 \%)$.

However the most common malignant lesions found in our study were metastatic lesions to the parotid gland "i.e. direct infiltration or distant metastasis" representing 28.57\%; while the most common primary parotid tumor was found to be lymphoma representing about $21.42 \%$ in all malignant lesions.

using conventional MRI alone in predicting benign and malignant lesions has the sensitivity of $68.75 \%$ and specificity of $66.67 \%$ with $78.57 \%$ positive predictive value and $54.55 \%$ negative predicative value. combining DWI and ADC values has increased accuracy, as the sensitivity and specificity were $92.86 \%$, $90.91 \%$ respectively with $92.86 \%$ positive predictive value and $90.91 \%$ negative predicative value

\section{Conclusion:}

The present study showed that ADC measurement has the potential to differentiate between benign and malignant focal parotid lesions.

The offered results for determining whether a parotid gland tumor is benign or malignant on the basis of combining morphologic features and DWIs \& ADC measurements in a diagnostic noninvasive approach are very promising and might decrease number of unnecessary invasive procedures e.g. biopsies or inappropriate extensive surgical approaches for benign parotid lesions. This is especially promising with regard to the differentiation of pleomorphic adenomas and Warthin tumors as well as carcinoma.

The lesions show the following characteristics on conventional MRI like high T2 signal, lobulated margin and heterogeneity as well as high ADC values are considered benign and mostly pleomorphic adenoma with no further need to biopsy. While lesions show ill-defined margins and low T2 signal, further characterized by DWIs and ADC values; if $\mathrm{ADC}$ is high, they mostly being benign and need further confirmation with FNAC or true cut biopsy. If the ADC is low they mostly malignant and no need for further histopathological evaluation.

The diffusion weighted imaging and ADC value give appreciable information about tumor cellularity with tissue contrast between the active and necrotic areas within the tumor. This would be valuable and could direct the beneficial site for interventional 
procedure and biopsy to be taken from the viable tumoural tissue especially in case with non-enhanced studies due to renal impairment).fig 32(

Conflict of interest:none of the contributors declared any conflict of interest

\section{References}

1. Alifa D, Khaled B, Kalil K, et al. Utility of diffusion-weighted MRI in distinguishing benign and malignant parotid lesions. ECR 2012/ C-2619.

2. Alifa D, Khaled B, Kalil K, et al. Utility of diffusion-weighted MRI in distinguishing benign and malignant parotid lesions. ECR 2012/ C-2619.

3. Aloudah NM, Raddaoui E, Aldhahri S, et al. Low-Grade

Papillary
Cystadenocarcinoma of the Parotid Gland: Presentation of a Case with Cytological, Histopathological and Immunohistochemical Features and Pertinent Literature Review. Diagnostic Cytopathology, 2008; 137 (2): $128-131$.

4. Andersson L, Bolling M, Wirestam R, et al. Combined diffusion weighting and CSF suppression in functional MRI. NMR Biomed 2002; 15: 235 - 240.

5. Andreadis D, Poulopoulos A, Epivatianos A, et al. Carcinosarcoma of the parotid gland: Immunohistochemical analysis with emphasis in cell cycle mitotic activity and cell adhesion molecules expression. Oral Oncology Extra 2006; 42: 140 - 143.

6. Arshad AR .Benign parotid lesions: is near total parotidectomy justified? Ann Acad Med Singapore 2006; 35: 889 - 891.

To cite this article: Medhat M. Rafat, Islam M. El- shazly, Christen h. mekhael Role of MRI Diffusion in Differentiation between Benign and Malignant Parotid Gland Tumors. BMFJ 2021;38 (2): 569-579. DOI: 10.21608/bmfj.2021.73364.1411 